\title{
Numerical distance effects in visual search
}

\author{
Wolf Schwarz • Anne-Kathrin Eiselt
}

Published online: 10 July 2012

(C) Psychonomic Society, Inc. 2012

\begin{abstract}
We present three experiments in which observers searched for a target digit among distractor digits in displays in which the mean numerical target-distractor distance was varied. Search speed and accuracy increased with numerical distance in both target-present and target-absent trials (Exp. 1A). In Experiment 1B, the target 5 was replaced with the letter S. The results suggest that the findings of Experiment $1 \mathrm{~A}$ do not simply reflect the fact that digits that were numerically closer to the target coincidentally also shared more physical features with it. In Experiment 2, the numerical distance effect increased with set size in both targetpresent and target-absent trials. These findings are consistent with the view that increasing numerical target-distractor distance affords faster nontarget rejection and target identification times. Recent neurobiological findings (e.g., Nieder, 2011) on the neuronal coding of numerosity have reported a width of tuning curves of numerosity-selective neurons that suggests graded, distance-dependent coactivation of the representations of adjacent numbers, which in visual search would make it harder to reject numerically closer distractors as nontargets.
\end{abstract}

Keywords Numerical distance effect · Visual search . Category effect $\cdot$ Mental number line $\cdot$ Numerical magnitude

W. Schwarz $(\bowtie)$

Department of Psychology, University of Potsdam,

P.O. Box 6015 53,

14415 Potsdam, Germany

e-mail: wschwarz@uni-potsdam.de

\section{A.-K. Eiselt}

Institute of Neurobiology, University of Tübingen,

Auf der Morgenstelle 28,

72076 Tübingen, Germany

e-mail: anne-kathrin.eiselt@biologie.uni-tuebingen.de
Numbers and numerical information play a central role in how we represent, communicate, and respond to quantity-related aspects of our environments. Correspondingly, a considerable amount of research has addressed many cognitive, neuronal, developmental, and clinical aspects of numerical cognition (for detailed recent reviews and discussion, see the volume edited by Dehaene \& Brannon, 2011).

At which functional stage does the quantity-related meaning of numbers begin to influence perceptual, central, or motor processes? The process of extracting numerical meaning is often associated with chronometrically later stages of information processing, such as response selection and activation. For example, in parity judgments, numerically small digits yield left-hand or left-foot responses, as well as left-directed saccades, that are relatively faster than the corresponding right-side responses, whereas for numerically large numbers, the opposite findings hold (Dehaene, Bossini, \& Giraux, 1993; Fischer, Warlop, Hill, \& Fias, 2004; Schwarz \& Keus, 2004; Schwarz \& Müller, 2006). The relatively late, motor-related electrophysiological correlates of this effect (Gevers, Ratinckx, de Baene, \& Fias, 2006; Keus, Jenks, \& Schwarz, 2005) and the results of behavioral studies based on the locus-of-slack diagnostic in a dual-task paradigm (Müller \& Schwarz, 2007) strongly suggest that this number-related compatibility effect arises at a relatively late processing stage, when the overt motor response is selected and prepared.

However, several results have suggested that, at least under favorable conditions, effects of numerical meaning can be observed that are usually related to earlier stages of processing. Fischer, Castel, Dodd, and Pratt (2003; also see Galfano, Rusconi, \& Umiltà, 2006) showed that in a simple detection task the presentation of a central task-irrelevant digit speeds up manual responses to visual signals in the left (digits 1,2) or the right (digits 8,9) visual hemifield, compatible with the view that numerically small (vs. large) digits automatically shift attention to the left (vs. the right) 
visual field. Corbett, Oriet, and Rensink (2006) found that numerical meaning can be extracted rapidly from visual displays so that perceptual comparisons between upright digits are made more quickly and accurately than comparisons with letters or sideways digits. Similar conclusions have been suggested by number-size interference experiments (Cohen Kadosh, Henik, \& Rubinsten, 2008; Schwarz \& Ischebeck, 2003), which indicated that judgments of the digit's physical size are systematically influenced by the digit's numerical meaning, leading to a pattern of mutual, bidirectional interference in the processing of number and (physical) size information. Related electrophysiological studies (e.g., Schwarz \& Heinze, 1998) have suggested that these interference effects arise during early stages of processing, with onsets well before $250 \mathrm{~ms}$. Finally, the numerical meaning of digits also influences judgments about the temporal order in which they are visually presented (Nicholls, Lew, Loetscher, \& Yates, 2011; Schwarz \& Eiselt, 2009), and Casarotti, Michielin, Zorzi, and Umiltà (2007) reported related effects when observers judged the temporal order of small peripheral dots with respect to central digits $(1,2$ vs. 8,9$)$.

Given this previous evidence, it seems important to know whether this pattern of findings extends to other wellinvestigated perceptual paradigms. One of the most basic perceptual tasks that we routinely face in daily life is to detect a potential target presented at a previously unknown location. In these situations, the background often consists of distractors whose similarity to the target is a major determinant of search efficiency. Studies of visual search typically define target-distractor similarity in terms of physical, nonsemantic dimensions such as color, form, orientation, size, and conjunctions of these features (for reviews, see Pashler, 1998, chap. 3; Wolfe \& Horowitz, 2004). The effect of semantically defined target-distractor relations is often studied by contrasting categories (e.g., letters vs. digits; Brand, 1971; Jonides \& Gleitman, 1972; but see Duncan, 1983; for more recent related results, see Hamilton, Mirkin, \& Polk, 2006; Smilek, Dixon, \& Merikle, 2006; Yang \& Zelinsky, 2009); a much-debated issue in this context is whether (or when) it is possible to guide attention toward specific categories. Pashler (1998, p. 144) concluded that the evidence he reviewed "suggests that semantic information can contribute to effective search," whereas Wolfe and Horowitz (2004) saw semantic categories as a "doubtful case" with regard to their ability to guide the deployment of attention.

These results and conclusions suggested the question addressed in the experiments described below: Do number-related target-distractor relations modify the efficiency with which humans search for a target digit in arrays of distractor digits? More specifically, we varied the mean numerical target-to-distractor distance in search displays consisting of digits, and we studied search efficiency as a function of numerical distance. Many current models of visual search (for a review, see Wolfe \& Horowitz, 2004) entail an initial preattentive stage that, together with topdown influences, creates a ranking of items in the order of their attentional priority. This information guides the selection of candidate items, which are processed up to a point at which they are rejected if they are distractors; the efficiency of this rejection mechanism is one major determinant of search speed. Extending classical behavioral results (Moyer \& Landauer, 1967) supporting the concept of a mental number line, neurobiological research (Nieder, 2005, 2011; Nieder, Freedman, \& Miller, 2002) on the width of the tuning curves of numerosity-selective neurons has suggested graded, distance-dependent coactivation that "smears" the internal representations of adjacent numbers. If the numerical magnitudes of the distractors are encoded and their distance from the target number influences how efficiently any given distractor can be rejected, then search speed should increase with increasing average target-distractor distance. Alternatively, if the assumed rejection mechanism precedes the extraction of specifically numberrelated semantic information, then the mean target-distractor distance should not systematically modify the efficiency of visual search.

\section{Experiments 1A and 1B}

The aims of Experiments 1A and 1B were (Exp. 1A) to test whether search efficiency in response-terminated displays made up of digits depends on the mean numerical targetdistractor distance and (Exp. 1B) to control for the effects of visual target-distractor similarity. We will first describe the Experiment 1A method.

\section{Method}

\section{Experiment $1 \mathrm{~A}$}

Participants A group of 36 paid University of Potsdam students, 18-26 years of age, with normal or corrected-tonormal vision participated in one session of approximately $1 \mathrm{~h}$.

Stimuli and apparatus Each search display contained 24 digits - eight at each of three eccentricities, positioned at one of the 12 clock positions along imaginary circles of radius $0.9,1.9$, and $3.5 \mathrm{deg}$. The actual position of each digit was randomly jittered away from the edge of the circle (at distances uniformly chosen between $\pm 6, \pm 20$, and $\pm 40 \mathrm{~min}$ from the three radii) so as to produce an irregular appearance but to avoid physical item overlap (see Fig. 1). The digits were presented in dark blue against a light gray 


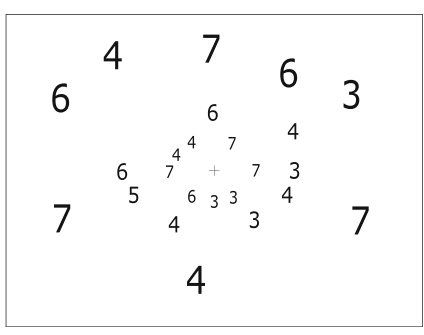

Fig. 1 All search displays in Exp. 1A contained 24 digits, two at each of the 12 possible clock positions, and eight at each of three eccentricities, jittered away from imaginary circles of radius $0.9,1.9$, and 3.5 deg. The example shows a close-context display where the target appears in the 8 o'clock position at the medium eccentricity. Added to the right for comparison are the targets 5, used in Experiment 1A, and $\mathrm{S}$, used in the control Experiment $1 \mathrm{~B}$

background in the proportional Verdana font using font heights that increased $(23,31$, and $52 \mathrm{~min})$ with eccentricity.

Procedure The task of the participants was to indicate, by pressing buttons with the middle or index finger of their preferred hand, whether or not the target digit 5 was present in the search display. Each trial started with the presentation of a red central fixation cross, followed after $300 \mathrm{~ms}$ by the search display. Following incorrect responses, error feedback was provided. Each of the six blocks contained 72 (half target-present and target-absent) trials. Three sets of numerical distractor contexts were used to generate the search displays: $\{3,4,6,7\}$ (close numerical distance, average 1.5); $\{2,3,7,8\}$ (medium distance, average 2.5); and $\{1,2,8,9\}$ (far distance, average 3.5).

\section{Experiment $1 B$}

This was run with 20 new, paid University of Potsdam students, 19-28 years of age, and was identical to Experiment 1A, with two exceptions. First, in target-present trials, the target digit 5 was replaced with the letter S (see Fig. 1); the task of the participants was to detect the presence or absence of the target S. Second, only the close and far numerical distance contexts were used.

\section{Results}

Experiment $1 \mathrm{~A}$ Error rates (as percentages) and median correct-response search times (RTs) for each participant were subjected to a repeated measures ANOVA with the factors Target (2: present/absent) and Distance (3: close/ medium/far).

As is shown in Fig. 2, mean RTs were about twice as long in target-absent $(1,796 \mathrm{~ms})$ as in target-present $(916 \mathrm{~ms})$ trials $\left[F(1,35)=236.14, p<.001, \eta^{2}=.87\right]$. More crucial to our study was the finding of a significant effect of numerical target-distractor distance, with decreasing RTs of 1,461 (close), 1,369 (medium), and 1,237 ms (far) $[F(2$, 70) $\left.=76.52, p<.001, \eta^{2}=.69\right]$.

Planned contrasts showed that for target-present trials, RTs were longer for close than for medium contexts $(p<.001)$, which in turn yielded longer RTs than did far contexts $(p<.05)$. For target-absent trials, RTs were shorter for far than for medium contexts $(p<.001)$, which in turn did not differ from close contexts $(p=.28)$. The two main effects interacted, indicating a larger distance effect for target-absent than for target-present trials $\left[F(2,70)=20.02, p<.001, \eta^{2}=.36\right]$. Given the decreasing error rates of $6.5,3.8$, and 3.4 for close, medium, and far contexts, respectively, these numerical distance effects cannot be explained on the basis of selective speed-accuracy trade-offs $\left[F(2,70)=32.95, p<.001, \eta^{2}=.48\right]$.

Experiment $1 B$ For target-present trials, the mean median RTs equaled $1,124 \mathrm{~ms}$ for the close-distance context and $1,123 \mathrm{~ms}$ for the far-distance context $[t(19)=0.04, p=.96]$; in target-absent trials, these means were 2,147 and $2,310 \mathrm{~ms}$ $\left[t(19)=5.01, p<.001, \eta^{2}=.57\right]$, respectively. The error rates for the close and far contexts were nearly identical, 4.7 and $4.5[t(19)=0.03, p=.97]$.

\section{Discussion}

Experiment $1 \mathrm{~A}$ indicated a significant and substantial decrease of search times with mean numerical target-distractor distance. This effect held for both target-present and target-absent trials, but it was larger under the latter condition, in accordance with standard findings in visual search (cf. Wolfe \& Horowitz, 2004). These results are consistent with the view that the degree of neuronal overlap of our mental representations of numbers influences the efficiency with which we search displays made up of digits. Descriptively, all individual comparisons are in line with this view, with only the difference between close and medium contexts in the target-absent condition not reaching significance. One potential explanation is that participants set an upper time limit (deadline) to terminate their search, which would necessarily selectively compress the mean RTs in these two conditions, which were by far the slowest.

There is a potential alternative account of our findings: namely, that search time and error rate decrease with numerical distance simply because the digits more distant from the target (5) might coincidentally share fewer physical features with the target than do those closer to it (cf. Duncan, 1983; Wolfe \& Horowitz, 2004). Several aspects of our results seem to argue against such an account, though. First, various "far" digits, such as 2 and 8 , seem physically more similar to the target 5 than do most "close" digits; indeed, the digits 2 and 5 are often chosen to illustrate visual similarity effects (e.g., Wolfe \& Horowitz, 2004, Fig. 1a). Second, if indeed a greater physical similarity of 5 to the numerically closer distractors could account for the distance effect found, a similar effect 
Fig. 2 Filled circles joined by lines show the mean median search times (correct responses only) for target-present (lower line) and target-absent (upper line) trials, as a function of the mean numerical target-distractor distance in Experiment 1A. Note the break in the left ordinate. Triangles joined by broken lines show the corresponding error rates in percentages, with the scale at the right ordinate. Error bars show \pm 2 SEs (Loftus \& Masson, 1994)

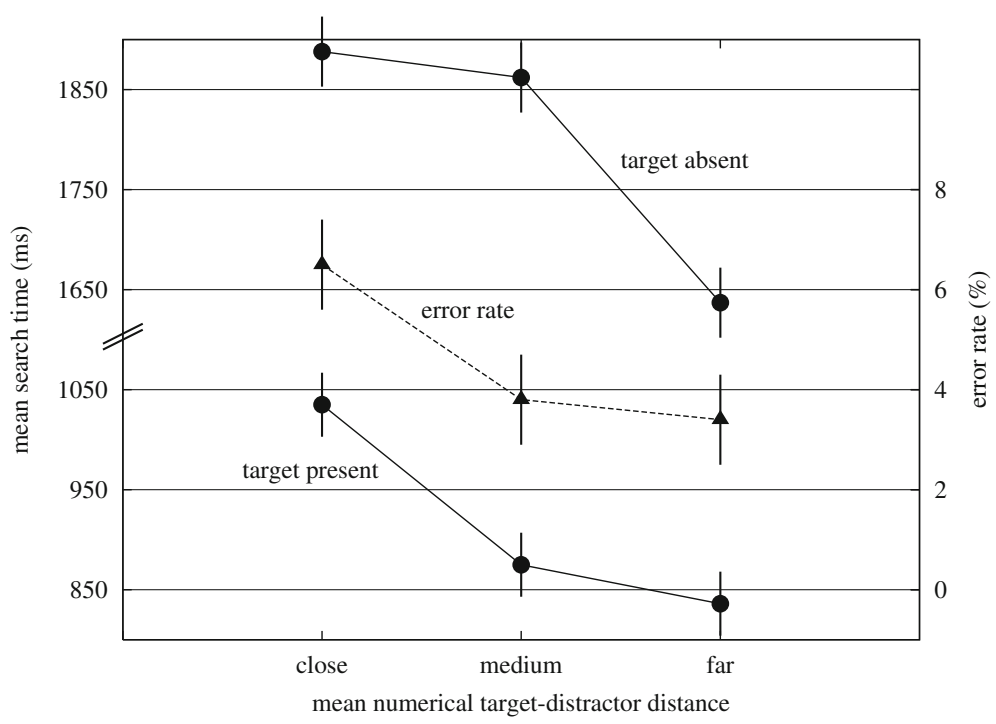

should presumably have been observed when searching for the target letter $\mathrm{S}$ among the same digit distractors as in Experiment 1A. The results of Experiment 1B do not support the view that the digits in the close context were more similar to $S$ (and thus to 5) than those of the far context; if anything, the target-absent results indicate the opposite. These results corroborate the view that the effect of numerical distance observed in Experiment 1A does not seem to simply reflect differences in physical similarity that are correlated with numerical targetdistractor distance.

\section{Experiment 2}

Which mechanism, or processing stage, benefits from increasing numerical distance? At least two functional loci seem plausible candidates and may be tentatively considered (Pashler, 1998; Wolfe \& Horowitz, 2004): a stage during which inspected distractors are rejected and the target is verified, or a decisional/strategic stage. The latter alternative refers to operations such as the decision to continue or terminate a hitherto unsuccessful search. According to the first alternative, numerical distance should affect the time required to compare the currently inspected items to the target. For example, the work of Nieder $(2005,2011)$ referred to above has suggested that the initial representation of numerosity is inherently fuzzy and induces a graded coactivation of adjacent numbers that might affect the precision and speed of these comparison and rejection processes.

One way to try to distinguish between these accounts would be to vary the set size of the displays and to study the interaction of this manipulation with numerical distance. More specifically, the frequency of distractor rejection processes necessarily increases in proportion to the set size. Therefore, if the first interpretation mentioned above is correct, we would expect that the numerical distance effect found in Experiment 1A should increase with set size. The second, "decisional" interpretation would also imply that search time would increase with set size, but it provides no basis to predict a Set Size $\times$ Numerical Distance interaction. The aim of Experiment 2 was to see whether the numerical target-distractor distance effect increases with display set size.

\section{Method}

Participants A group of 36 University of Otago students (25 female, 18-37 years of age) with normal or corrected-tonormal vision participated for course credit in one session of approximately $1 \mathrm{~h}$.

Stimuli, apparatus, and procedure All aspects were identical to their counterparts in Experiment 1A, with two exceptions. First, the display set size now equaled 12, 18, or 24 items, equally balanced across the three eccentricities and the 12 clock positions (for set size 18, six clock positions contained one, and six other positions two items). Second, only the close and far numerical distance contexts were used. Again, each of six blocks consisted of 72 trials, six for each combination of the factors Target (present/absent), Distractors (close/far), and Set Size (12/18/24).

Results and discussion

As is shown in Fig. 3, all three factors had clear main effects: Search times were longer in target-absent $(1,392 \mathrm{~ms})$ than in target-present $(780 \mathrm{~ms})$ trials $\left[F(1,35)=137.13, p<.001, \eta^{2}=\right.$ $.80]$, were longer in the close $(1,183 \mathrm{~ms})$ than in the far (989 ms) context $\left[F(1,35)=103.11, p<.001, \eta^{2}=.75\right]$, and increased with set size $\left[F(2,70)=129.34, p<.001, \eta^{2}=.79\right]$. Furthermore, all three simple interactions were significant: The 


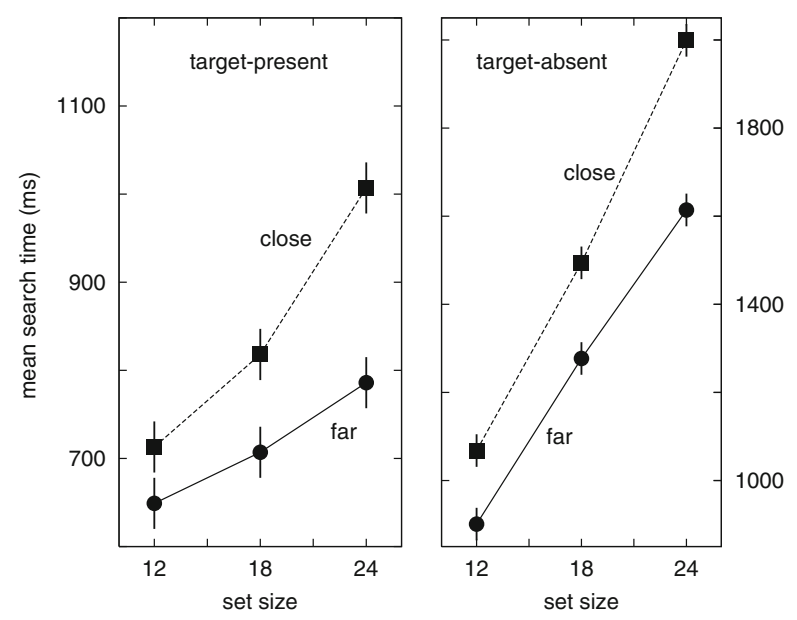

Fig. 3 Left panel: Target-present data. Filled circles joined by solid lines show the mean median search times (RTs, correct responses only) for the far numerical context. RTs for close numerical context are shown by squares and broken lines. Right panel: Target-absent data, in the same format as the target-present data. Note that the vertical axes of the two panels are scaled in a ratio of 2:1. Error bars show \pm 2 SEMs (Loftus \& Masson, 1994)

set size slope was steeper for target-absent $(68 \mathrm{~ms} /$ item $)$ than for target-present $(20 \mathrm{~ms} /$ item $)$ trials $[F(2,70)=$ 113.00, $\left.p<.001, \eta^{2}=.76\right]$. Replicating Experiment 1A, the effect of numerical distance was larger for targetabsent $(256 \mathrm{~ms})$ than for target-present $(132 \mathrm{~ms})$ trials $\left[F(1,35)=16.32, p<.001, \eta^{2}=.32\right]$. And, of greatest importance in the present context was the significant increase of the distance effect (from 116, through 164, to $303 \mathrm{~ms})$ with set size $\left[F(2,70)=36.36, p<.001, \eta^{2}=\right.$ $.51]$. As Fig. 3 shows, this Distance $\times$ Set Size interaction was found individually for both target-present $[F(2,70)=$ 15.79, $\left.p<.001, \eta^{2}=.31\right]$ and target-absent $[F(2,70)=$ $\left.18.92, p<.001, \eta^{2}=.35\right]$ trials; the three-way interaction was not significant $[F(2,70)=1.02, p=.37]$.

The pattern of error rates (in percentages) complements these RT findings. As is typical of visual search, false alarms were rare $(M=0.7)$, and 28 participants committed at most 2. Misses $(M=6.0)$ were more common, as well as being significantly more frequent for the close (8.1) than for the far (3.9) context $\left[F(1,35)=37.68, p<.001, \eta^{2}=.52\right]$. The difference in error rates between the close and far contexts was constant or increased with set size [3.1, 2.9, and 6.4, respectively; $F(2,70)=4.67, p<.012, \eta^{2}=.12$ ], so that the corresponding RT findings cannot be attributed to selective speed-accuracy trade-offs.

\section{General discussion}

Does symbolic numerical information influence how efficiently human observers search for a target digit (5) among distractor digits? Note that even an observer ignorant of the usual conventions of coding numerosity symbolically by digits could have easily participated in our experiments with a low error rate, using a strategy of purely physical feature analysis, without any activation of number-related semantic associations. Experiment 1A revealed a reliable performance benefit, in terms of both speed and accuracy, with increasing numerical target-distractor distance. The results of control Experiment 1B make it implausible that this performance benefit could be attributed to a coincidentally greater physical target similarity of those distractors that were numerically close to the target. The results of Experiment 2, and in particular the interaction of numerical distance with set size effects, suggest that the effect is unlikely to arise at a stage at which strategic decisions about resampling the search display and terminating the search process are made. In line with previous results on the width of neuronal numerosity tuning curves (Nieder, 2005, 2011), our results suggest that the items of our search displays are internally represented in a noisy format in which representational overlap increases with decreasing numerical distance. This "smearing" mechanism of distance-dependent graded coactivation of adjacent numbers necessitates an extended neuronal sampling period in order to improve the signal-to-noise ratio up to an acceptable accuracy level, ultimately leading to longer nontarget rejection and target verification times.

The difference in time required for comparisons of close $(4,6)$ versus far $(1,9$ or 2,8$)$ distractors to the target digit 5 is typically estimated to be approximately $25 \mathrm{~ms}$ (Dehaene, 1996; Schwarz \& Ischebeck, 2000). If the number of distractor-target comparisons in any one trial increases in proportion to set size, or when the target is absent, the overall numerical distance effect would roughly correspond to the present findings. Similar numerical distance effects have previously been reported by DeRosa and Morin (1970) for memory rather than visual search. Clearly, physical target-distractor similarity can influence and modify these distance effects (but see Exp. 1B), so an effective control experiment could compare groups of participants who did or did not know the numerical meanings of the characters used to generate the search displays. While Arabic digits are now nearly universally known, search among, for instance, Hindi or Thai number symbols could be compared between participants who did or did not know their meaning. Our results are reminiscent of more traditional category effects in visual search (Hamilton et al., 2006; Smilek et al., 2006; Yang \& Zelinsky, 2009), but differ from such effects in that they reflect the graded within-category influence of symbolic distance among items that are all elements of the same category. Our findings fit in well with other results (Casarotti et al., 2007; Corbett et al., 2006; Fischer et al. 2003) suggesting that symbolic numerical meaning is extracted rapidly from visual displays containing digits, and under favorable conditions, may influence basic perceptual tasks such as visual search. 
Author Note Part of this work was presented at the 51. Tagung experimentell arbeitender Psychologen (TeaP), Jena, Germany, held from March 29 to April 1, 2009. We thank Jeff Miller and Peter Shepherdson at the University of Otago, New Zealand, for support in conducting Experiment 2, and for helpful suggestions and discussions regarding the present work.

\section{References}

Brand, J. (1971). Classification without identification in visual search. Quarterly Journal of Experimental Psychology, 23, 178-186.

Casarotti, M., Michielin, M., Zorzi, M., \& Umiltà, C. (2007). Temporal order judgment reveals how number magnitude affects visuospatial attention. Cognition, 102, 101-117.

Cohen Kadosh, R., Henik, A., \& Rubinsten, O. (2008). Are Arabic and verbal numbers processed in different ways? Journal of Experimental Psychology: Learning, Memory, \& Cognition, 34, 1377-1391. doi:10.1037/a0013413

Corbett, J. E., Oriet, C., \& Rensink, R. A. (2006). The rapid extraction of numeric meaning. Vision Research, 46, 1559-1573.

Dehaene, S. (1996). The organization of brain activations in number comparison: Event-related potentials and the additive-factors method. Journal of Cognitive Neuroscience, 8, 47-68.

Dehaene, S., Bossini, S., \& Giraux, P. (1993). The mental representation of parity and number magnitude. Journal of Experimental Psychology: General, 122, 371-396. doi:10.1037/0096-3445.122.3.371

Dehaene, S., \& Brannon, E. M. (Eds.). (2011). Space, time and number in the brain: Searching for the foundations of mathematical thought. San Diego: Academic.

DeRosa, D. V., \& Morin, R. E. (1970). Recognition reaction time for digits in consecutive and nonconsecutive memorized sets. Journal of Experimental Psychology, 83, 472-479.

Duncan, J. (1983). Category effects in visual search: A failure to replicate the "oh-zero" phenomenon. Perception \& Psychophysics, 34, 221232. doi:10.3758/BF03202949

Fischer, M. H., Castel, A. D., Dodd, M. D., \& Pratt, J. (2003). Perceiving numbers causes spatial shifts of attention. Nature Neuroscience, 6, 555-556.

Fischer, M. H., Warlop, N., Hill, R. L., \& Fias, W. (2004). Oculomotor bias induced by number perception. Experimental Psychology, 51, 91-97.

Galfano, G., Rusconi, E., \& Umiltà, C. (2006). Number magnitude orients attention, but not against one's will. Psychonomic Bulletin \& Review, 13, 869-874.

Gevers, W., Ratinckx, E., De Baene, W., \& Fias, W. (2006). Further evidence that the SNARC effect is processed along a dual-route architecture: Evidence from the lateralized readiness potential. Experimental Psychology, 53, 58-68. doi:10.1027/1618-3169.53.1.58

Hamilton, J. P., Mirkin, M., \& Polk, T. A. (2006). Category-level contributions to the alphanumeric category effect in visual search. Psychonomic Bulletin \& Review, 13, 1074-1077. doi:10.3758/ BF03213928

Jonides, J., \& Gleitman, H. (1972). Conceptual category effect in visual search: $\mathrm{O}$ as letter or as digit. Perception \& Psychophysics, $12,457-460$
Keus, I. M., Jenks, C., \& Schwarz, W. (2005). Psychophysiological evidence that the SNARC effect has a functional locus in a response selection stage. Cognitive Brain Research, 24, 48-56.

Loftus, G. R., \& Masson, M. E. J. (1994). Using confidence intervals in within-subject designs. Psychonomic Bulletin \& Review, 1, 476-490. doi:10.3758/BF03210951

Moyer, R. S., \& Landauer, T. K. (1967). Time required for judgements of numerical inequality. Nature, 215, 1519-1520. doi:10.1038/ $2151519 \mathrm{a} 0$

Müller, D., \& Schwarz, W. (2007). Exploring the mental number line: Evidence from a dual-task paradigm. Psychological Research, 71, 598-613.

Nicholls, M. E. R., Lew, M., Loetscher, T., \& Yates, M. J. (2011). The importance of response type to the relationship between temporal order and numerical magnitude. Attention, Perception, \& Psychophysics, 73, 1604-1613.

Nieder, A. (2005). Counting on neurons: The neurobiology of numerical competence. Nature Reviews Neuroscience, 6, 177-190.

Nieder, A. (2011). The neural code for number. In S. Dehaene \& E. M. Brannon (Eds.), Space, time and number in the brain: Searching for the foundations of mathematical thought (pp. 103-118). San Diego, CA: Academic Press.

Nieder, A., Freedman, D. J., \& Miller, E. K. (2002). Representation of the quantity of visual items in the primate prefrontal cortex. Science, 297, 1708-1711. doi:10.1126/science.1072493

Pashler, H. (1998). The psychology of attention. Cambridge: MIT Press.

Schwarz, W., \& Eiselt, A. K. (2009). The perception of temporal order along the mental number line. Journal of Experimental Psychology: Human Perception and Performance, 35, 989-1004.

Schwarz, W., \& Heinze, H.-J. (1998). On the interaction of numerical and size comparison: A behavioral and event-related potential study. Neuropsychologia, 36, 1167-1179.

Schwarz, W., \& Ischebeck, A. (2000). Sequential effects in number comparison. Journal of Experimental Psychology: Human Perception and Performance, 26, 1606-1621.

Schwarz, W., \& Ischebeck, A. (2003). On the relative speed account of number-size interference effects in comparative judgments of numerals. Journal of Experimental Psychology: Human Perception and Performance, 29, 507-522.

Schwarz, W., \& Keus, I. M. (2004). Moving the eyes along the mental number line: Comparing SNARC effects with saccadic and manual responses. Perception \& Psychophysics, 66, 651-664. doi:10.3758/BF03194909

Schwarz, W., \& Müller, D. (2006). Spatial associations in numberrelated tasks: A comparison of manual and pedal responses. Experimental Psychology, 53, 4-15.

Smilek, D., Dixon, M. J., \& Merikle, P. M. (2006). Revisiting the category effect: The influence of meaning and search strategy on the efficiency of visual search. Brain Research, 1080, 73-90.

Wolfe, J. M., \& Horowitz, T. S. (2004). What attributes guide the deployment of visual attention and how do they do it? Nature Reviews Neuroscience, 5, 495-501. doi:10.1038/nrn1411

Yang, H., \& Zelinsky, G. J. (2009). Visual search is guided to categorically-defined targets. Vision Research, 49, 2095-2103. doi:10.1016/j.visres.2009.05.017 